ARTICLE

\title{
Ultra-rapid and highly efficient enrichment of organic pollutants via magnetic mesoporous nanosponge for ultrasensitive nanosensors
}

\author{
Lingling Zhang (10 1,5, Yu Guo ${ }^{1,5}$, Rui Hao ${ }^{1}$, Yafei Shi ${ }^{1}$, Hongjun You (1) ${ }^{2}$, Hu Nan ${ }^{3}$, Yanzhu Dai ${ }^{3}$, Danjun Liu ${ }^{4}$,
} Dangyuan Lei ${ }^{4} \&$ Jixiang Fang (1) ${ }^{1,2 凶}$

Currently, owing to the single-molecule-level sensitivity and highly informative spectroscopic characteristics, surface-enhanced Raman scattering (SERS) is regarded as the most direct and effective detection technique. However, SERS still faces several challenges in its practical applications, such as the complex matrix interferences, and low sensitivity to the molecules of intrinsic small cross-sections or weak affinity to the surface of metals. Here, we show an enrichment-typed sensing strategy with both excellent selectivity and ultrahigh detection sensitivity based on a powerful porous composite material, called mesoporous nanosponge. The nanosponge consists of porous $\beta$-cyclodextrin polymers immobilized with magnetic NPs, demonstrating remarkable capability of effective and fast removal of organic micropollutants, e.g., $\sim 90 \%$ removal efficiency within $\sim 1 \mathrm{~min}$, and an enrichment factor up to $\sim 10^{3}$. By means of this current enrichment strategy, the limit of detection for typical organic pollutants can be significantly improved by 2 3 orders of magnitude. Consequently, the current enrichment strategy is proved to be applicable in a variety of fields for portable and fast detection, such as Raman and fluorescent sensing.

\footnotetext{
${ }^{1}$ Key Laboratory of Physical Electronics and Devices of Ministry of Education, School of Electronic Science and Engineering, Faculty of Electronic and Information Engineering, Xi'an Jiaotong University, Xi'an, Shaanxi 710049, China. ${ }^{2}$ Key Laboratory of Biomedical Information Engineering of Ministry of Education, School of Life Science and Technology, Xi'an Jiaotong University, Xi'an, Shaanxi 710049, China. ${ }^{3}$ School of Microelectronics, Faculty of Electronic and Information Engineering, Xi'an Jiaotong University, Xi'an Jiaotong University, Xi'an, Shaanxi 710049, China. ${ }^{4}$ Department of Materials Science and Engineering, City University of Hong Kong, 83 Tat Chee Avenue, Kowloon 999077 Hong Kong, China. ${ }^{5}$ These authors contributed equally: Lingling Zhang, Yu Guo. ${ }_{\text {email: jxfang@mail.xjtu.edu.cn }}$
} 


\section{T} he Stockholm Convention on Persistent Organic Pollutants (POPs) was endorsed by 131 nations in 2004 to eliminate the most persistent bioaccumulative and toxic substances in the world ${ }^{1}$. Organic micropollutants of ground and surface water resources, such as pesticides and plastic components, have aroused great concerns about potential negative effects on aquatic ecosystems and human health ${ }^{2,3}$. Therefore, parallel to the researches of adsorbent materials to remove organic pollutants from water, the ultrasensitive detection of organic pollutants is another crucial field, since the solubility of organic micropollutants in water is always at the trace level ${ }^{4}$. Among diverse detection approaches, surface-enhanced Raman spectroscopy (SERS), achieved breakthroughs in 1997 and became the first vibrational spectroscopy technique that could provide delicate information on molecular fingerprints with a potential of singlemolecule sensitivity ${ }^{5-9}$, thus regarded as the most simple, fast, flexible, and portable detection technique.

However, it seems surprising that after fifty decades, SERS has not yet been widely used in practical applications ${ }^{10}$. This is owing to the fact that, besides the stability of SERS substrates and reproducibility of spot-to-spot, SERS still faces two major bottlenecks in the commercial market. The first is the low detection sensitivity to the molecules of intrinsic small cross-sections or weak affinity to metal surface. As we know, SERS is an optical near-field effect ${ }^{11-13}$. A high activity can be obtained only when the target molecule is very close to the plasmonic surface. Therefore, researchers exploited diverse approaches to capture the target molecules onto the metal surface by means of antibodies, aptamers, ion liangs, et al. ${ }^{14,15}$. However, the ultra-rapid capture to meet on-site and portable detection remains a challenge. The second is the interference from the complex matrices ${ }^{16}$. In real-sample detection, most organic pollutants in water cannot be effectively adsorbed onto the metallic surface because of their low affinity toward the metal, hence the metal surface is usually inactivated due to unspecific adsorption by the interference from matrix molecules in the surrounding environment. Thus, for commercial applications, an ultra-fast and effective pretreatment is of importance to eliminate the most matrix interference. Therefore, recently, some strategies, e.g., selective separation, concentration, enrichment from complex matrix, and spatial localization of target molecules ${ }^{17-19}$, are suggested to solve this long-standing challenge.

In this work, we propose a new sensing strategy in rapid separation and highly efficient enrichment of POPs from complicated real-sample matrices by means of the magnetic NPs immobilized porous $\beta$-CD polymer (MN-PCDP), called mesoporous nanosponge. The current strategy (the schematic description of the protocol is shown in Fig. 1) demonstrates several remarkable advantages. Firstly, specific and selective absorption and separation of target molecules eliminate the matrix interference. When the MN-PCDP adsorbent is dispersed into the water in the beaker containing organic pollutants and impurities (shown in Fig. 1a, b), specific and selective adsorption of target molecules can be achieved. In fact, microporous $\beta-C D$ material has been widely studied because of its outstanding adsorption efficiency through forming host-guest inclusion with many hydrophobic organic pollutants ${ }^{20,21}$. The magnetic NPs are introduced into the MN-PCDP compounds to rapidly separate the adsorbent from water, thus eliminates the interference of unspecific adsorption from the complex matrix in the realsamples. Secondly, highly efficient concentration and enrichment capability thus ultra-high detection sensitivity can be obtained. The MN-PCDP adsorbent demonstrates a remarkable removal efficiency on organic micropollutants, e.g., 90\% (relative standard deviation, $\mathrm{RSD}<1 \%)$. Meanwhile, the adsorbed pollutants from initial water of $\sim 1000 \mathrm{ml}$ can be desorbed in ethanol with a volume of $\sim 1 \mathrm{ml}$ (Fig. 1c), for further analysis such as UV-vis, Raman, and fluorescent spectroscopy. Thus, an ultra-high enrichment efficiency with an enrichment factor up to $\sim 10^{3}$ times can be obtained (RSD $<5 \%$ ), and the limit of detection (LOD) in a variety of sensing applications can be lowered by $2-3$ orders of magnitude. Thirdly, ultra-quick enrichment processes thus on-site portable detection can be realized. In the current strategy, ultra-fast adsorption, magnetic separation, and desorption can be accomplished, i.e., totally within $2-3 \mathrm{~min}$. Thus, the current sensing strategy can be believed to be applicable to a wider range of sensing areas for an economical, simple, fast, flexible, and portable detection.

\section{Results}

Synthesis and characterization of MN-PCDP nanosponges. The microporous MN-PCDP material, an inexpensive and renewable carbohydrate, which is featured by small pores and high surface areas, is used in this work as an excellent adsorbent. The MNPCDP is prepared by cross-linking polymerization of $\beta-C D$ and cross-linking agent (tetrafluoroterephthalonitrile (TFT)), with magnetic NPs $\left(\mathrm{Fe}_{3} \mathrm{O}_{4}\right)$ in one-step solvothermal reaction. Supplementary Fig. $1 \mathrm{a}-\mathrm{c}$ shows the transmission electron microscope (TEM) images of magnetic NPs $\left(\mathrm{MN}, \mathrm{Fe}_{3} \mathrm{O}_{4}\right)$, porous $\beta$-CD polymer (PCDP), and MN-PCDP, respectively. As shown in Supplementary Fig. 1a, the synthesized $M N$ exhibits regular spheres with good dispersibility and uniform size (average size $\sim 200 \mathrm{~nm}$ ). Supplementary Fig. 1b exhibits that the PCDP is a porous network structure. After the immobilization of $\mathrm{MN}$, as shown in Supplementary Fig. 1c, the porous network structure of MN-PCDP is not disrupted. The Fourier transform-infrared

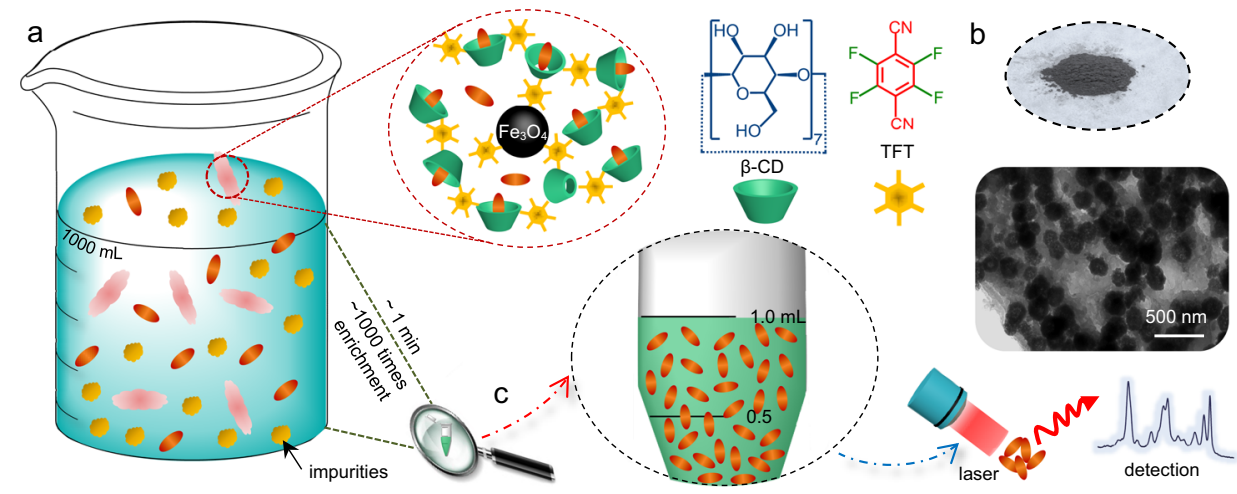

Fig. 1 Schematic of the current enrichment and detection based on the porous $\boldsymbol{\beta}$-CD polymer. a Adsorption and $\mathbf{c}$ desorption processes using magnetic NPs immobilized porous $\beta-C D$ polymer (MN-PCDP) with 1000 times enrichment. b Optical photograph and TEM imagine of MN-PCDP. 
spectroscopy (FT-IR) spectra of MN, $\beta$-CD, TFT, PCDP, MNPCDP are displayed in Supplementary Fig. 1d. The absorption bands at 1652 and $1396 \mathrm{~cm}^{-1}$ of the MN can be associated with carboxylate group ${ }^{22}$. The FT-IR spectrum of the MN-PCDP obviously combines the characteristic peaks of the TFT and the $\beta$ $\mathrm{CD}$. The signal intensity of absorption peak at $1265 \mathrm{~cm}^{-1}$ in relation to C-F stretching vibration is weaker than that in TFT owing to the partial replacement of $\mathrm{F}^{23,24}$, implying that the $\beta$ - $\mathrm{CD}$ has been crosslinked with TFT (Supplementary Fig. 2). Supplementary Figure 1e indicates that the Brunauer-Emmett-Teller surface areas $\left(S_{\mathrm{BET}}\right)$ of MN-PCDP is about $66 \mathrm{~m}^{2} \mathrm{~g}^{-1}$. The pores with diameter of $1.7-3.0 \mathrm{~nm}$ comprise the majority of the free volume of MN-PCDP and its average pore diameter is $\sim 2.12 \mathrm{~nm}$.

Adsorption behavior of MN-PCDP nanosponges. The high surface area and permanent porosity of MN-PCDP mesoporous nanosponge enable the rapid removal of organic micropollutants from water ${ }^{25}$. As shown in Supplementary Fig. 3, the PCDP and MN-PCDP display almost the same properties in time-dependent adsorptions of bisphenol A (BPA), revealing the immobilization of magnetic NPs has no remarkable influence on the adsorption performance of PCDP. The time-dependent adsorptions of various organic micropollutants adsorbed by MN-PCDP, including plastic components, pesticide, and aromatic model compounds (Fig. 2a), are shown in Fig. 2b, Supplementary Fig. 4, and Supplementary Table 1. Each organic micropollutant is rapidly removed, reaching $\sim 95 \%$ of its equilibrium uptake in $10 \mathrm{~s}^{20}$. The removal efficiencies of BPA, parathion, carbendazim, and 2-naphthol (2-NO) by MN-PCDP are more than $80 \%$ in $30 \mathrm{~s}$, which is much higher than the Norit ROW 0.8 supra extruded activated carbon (NAC) as presented in Fig. 2c, Supplementary Fig. 5, 6 and Supplementary Table 2. In this work, different crosslinking agent, e.g., epichlorohydrin (EPI) is compared
(Supplementary Fig. 7). As shown in Supplementary Fig. 8, the removal efficiency of BPA by MNEPI-CDP in $1 \mathrm{~min}$ is $19.5 \%$, which is much lower than MN-PCDP. We further probe the readily accessible binding sites of MN-PCDP by determining the flow-through uptake of different organic micropollutants. In these experiments, the adsorbent $(\sim 5 \mathrm{mg})$ is trapped as a thin layer on a $0.22 \mu \mathrm{m}$ syringe filter, and aqueous organic pollutants $(5 \mathrm{~mL}, 0.1 \mathrm{mM})$ passed rapidly through the filter at a flow rate of $10 \mathrm{ml} \mathrm{min}^{-1}$ (Supplementary Fig. 9). Under these conditions, for example, $76 \%$ of the BPA is removed from the solution, corresponding to more than $84 \%$ of its equilibrium adsorption, confirming that the host-guest interaction plays a major role in the filtration process by syringe ${ }^{26}$.

As is known, the hydroxyl groups of $\beta$-CD are located at the outer surface of the molecule, that is, primary hydroxyls at the narrow side and secondary hydroxyls at the wider side, which makes $\beta$-CD water-soluble but simultaneously generates an inner cavity that is relatively hydrophobic ${ }^{27}$. Because of their hydrophobic interior cavity, $\beta$-CD can either partially or entirely accommodate suitably sized lipophilic low molecular weight molecules or even polymers ${ }^{28}$. The superior performance of MNPCDP can be helpful to that its $\beta-C D$ moieties are easily accessed by most of the organic micropollutants, and these molecules can be rapidly trapped in the cavity of $\beta$-CD. For example, MN-PCDP exhibits a remarkable adsorption capability and selectivity for most aromatics and some chain compounds, as shown in Fig. $2 \mathrm{~b}$ and Supplementary Figs. 10, 11. Furthermore, by means of particular treatments such as changing $\mathrm{pH}$ value of solution ${ }^{29}$, the adsorption feature of molecules can be tuned. Thus, the MNPCDP mesoporous nanosponge will display a wide applicability and selectivity in a variety of molecules.

Furthermore, the influence of the concentrations of adsorbent on the adsorption efficiency of BPA is studied and shown in
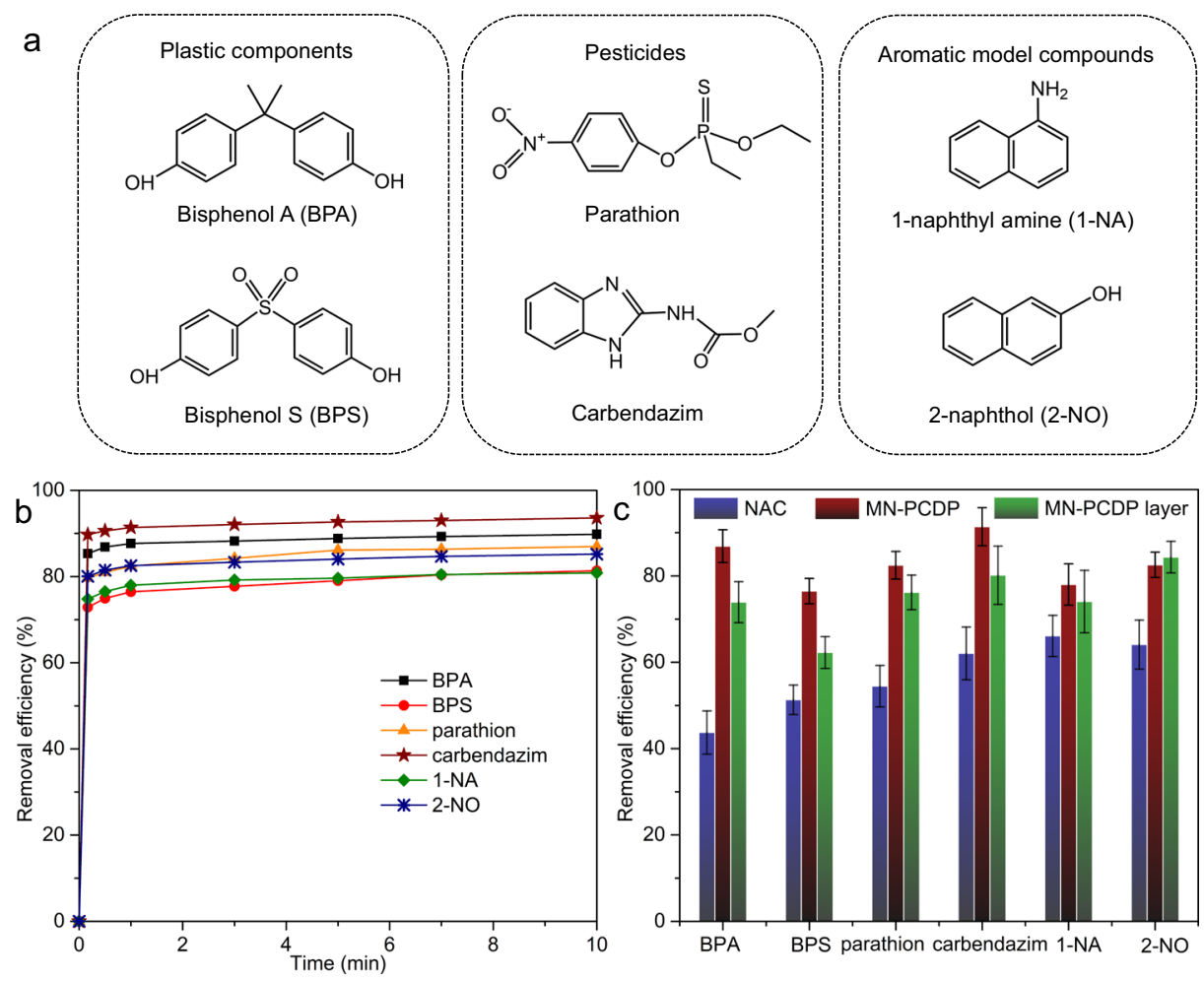

Fig. 2 MN-PCDP rapidly adsorbs a broad range of organic pollutants. a Structures of each tested organic pollutant. $\mathbf{b}$ Time-dependent adsorption of each pollutant $(0.1 \mathrm{mM})$ by MN-PCDP $\left(1 \mathrm{mg} \mathrm{mL}^{-1}\right)$. c Percentage removal efficiency of each pollutant obtained by stirring NAC (blue), stirring MN-PCDP (red), and rapidly flowing through a thin MN-PCDP layer (green). The data are reported as the average uptake of triplicate experiments. Error bars mean standard deviations 
a
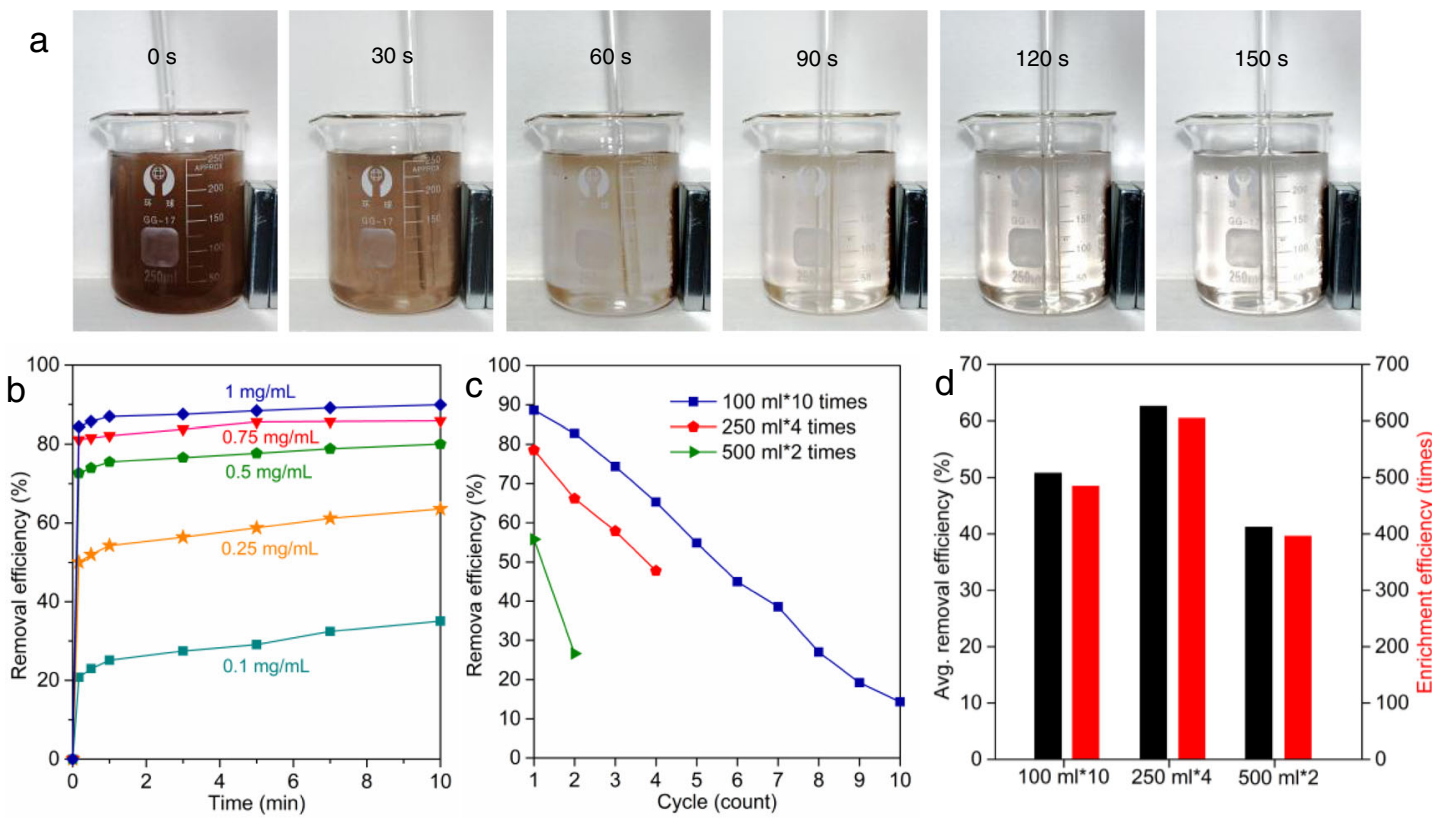

Fig. 3 Rapid enrichment performance of MN-PCDP. a Optical photographs of MN-PCDP separation process by a magnet in continuous time. $\mathbf{b}$ Timedependent adsorption of BPA $(0.1 \mathrm{mM})$ using MN-PCDP with different dosage $\left(0.1,0.25,0.5,0.75\right.$, and $\left.1 \mathrm{mg} \mathrm{L}^{-1}\right)$. c Removal efficiency of BPA (0.01 mM) using MN-PCDP (100 mg) in three methods ( $100 \mathrm{~mL}$ for 10 times, $250 \mathrm{~mL}$ for 4 times and $500 \mathrm{~mL}$ for 2 times). d Average removal (black) and enrichment (red) efficiency of the three methods in (c).

Fig. 3b, Supplementary Fig. 12, and Supplementary Table 3. When the concentration increases from 0.1 to $1.0 \mathrm{mg} \mathrm{L}^{-1}$, the adsorption efficiency of BPA is enhanced from 25.12 to $87.09 \%$ within $1 \mathrm{~min}$ and from 35.07 to $89.82 \%$ within $10 \mathrm{~min}$.

Desorption, concentration, and enrichment features of $\mathrm{MN}$ PCDP nanosponges. As we all know, organic micropollutants exhibit good solubility in organic solution, such as ethanol and methanol ${ }^{20}$. Hence, after adsorption process, we may separate the MN-PCDP mesoporous nanosponges from the solution quickly by the magnet, and utilize ethanol to desorb the organic micropollutants from the adsorbent, thus obtain the enriched pollutant solution. In order to obtain higher concentration of desorbed micropollutant solution, in this work, we chose $1 \mathrm{~mL}$ ethanol to desorb organic micropollutants adsorbed in MN-PCDP adsorbent. As shown in Supplementary Fig. 13, the concentration of BPA can be increased to 88.5 times of its initial concentration with a recipe of $100 \mathrm{~mL}$ organic pollutant (BPA) solution and $100 \mathrm{mg}$ MN-PCDP adsorbent. This result reveals that more than $98 \%$ of the adsorbed organic micropollutants are desorbed into the ethanol solution. As discussed in Fig. 3b, with the concentration of adsorbent increases, the adsorption efficiency tends to reach equilibrium. Considering the cost increase of sample preparation and dosage of adsorbent in the desorption process (with $1 \mathrm{~mL}$ ethanol), $20-100 \mathrm{mg} / 100 \mathrm{~mL}$ of adsorbent is selected as the adsorbent concentration in subsequent experiments.

In order to further improve the enrichment efficiency of $100 \mathrm{mg}$ adsorbent in total $1000 \mathrm{~mL}$ organic micropollutants, herein, we attempted three methods during the adsorption and desorption processes, including $100 \mathrm{~mL} \times 10$ times, $250 \mathrm{~mL} \times 4$ times, and $500 \mathrm{~mL} \times 2$ times. Importantly, the adsorbent can be simply separated by a magnet in every adsorption cycle, then desorbed in ethanol. As shown in Fig. 3c, Supplementary Fig. 14, and Supplementary Table 4, as the recycling adsorption times increase, the removal efficiencies of these three methods gradually decrease. The average removal efficiencies in methods of $100 \mathrm{~mL}$ $\times 10$ times, $250 \mathrm{~mL} \times 4$ times, and $500 \mathrm{~mL} \times 2$ times are 50.98 ,
62.58 , and $41.22 \%$, respectively. These results represent an enrichment capability of 485, 605, and 396 times of the initial concentration (Fig. 3d), respectively. Thus, we achieve a notable enrichment factor of above 600 times of initial organic pollutants via $1000 \mathrm{~mL}$ initial solution by means of the optimization of adsorption and desorption processes. Here, the optimized parameters, i.e., $100 \mathrm{mg}$ adsorbent in $250 \mathrm{~mL} \times 4$ cycle times, are used for the succedent experiments. Meanwhile, it is also worth pointing out that the separation process by a magnet is very fast and facile, such as $100 \mathrm{~mL}$ with $60 \mathrm{~s}$ (Supplementary Fig. 15a), $250 \mathrm{~mL}$ with $90 \mathrm{~s}$ (Fig. 3a and Supplementary Fig. 15b), and $500 \mathrm{~mL}$ with $150 \mathrm{~s}$ (Supplementary Fig. 15c). Therefore, the current ultra-fast enrichment protocol may fully meet the requirement of on-site and portable detection applications.

SERS and fluorescence measurements of pollutants via current enrichment protocol. In order to evaluate the advantage of the current enrichment protocol on the detection sensitivity, fluorescence, and SERS spectra of five POPs molecules, e.g., BPA, carbendazim, tetramethyl thiuram disulfide (TMTD, thiram), diquat, and anthracene, are measured using MN-PCDP nanosponges as adsorbent. The hydrophobic slippery SERS platform ${ }^{30}$ with $\sim 55 \mathrm{~nm}$ Au NPs (Supplementary Fig. 16) is adopted for the measurement of SERS spectra. As shown in Fig. 4a, b, without the enrichment process, the LOD of SERS for TMTD is around $1 \mathrm{pM}$. However, after the enrichment using MN-PCDP adsorbent, this value reaches to $\sim 5 \mathrm{fM}$, showing an increase of $10^{2}-10^{3}$. Meanwhile, based on this enrichment protocol, the LOD of BPA, carbendazim, diquat and anthracene are up to $0.1 \mathrm{nM}, 5 \mathrm{pM}, 1$ $\mathrm{pM}$, and $1 \mathrm{nM}$ (Supplementary Figs. 17-20), which are much lower than most of the magnetic SERS-based sensors (Table 1). Furthermore, multiple adsorption and desorption experiments by MN-PCDP for the above five organic molecules are implemented to illustrate the reproducibility of this adsorbent. In Supplementary Figs. 21-23 and Supplementary Tables 5, 6, the removal efficiencies and enrichment efficiencies of MN-PCDP adsorbent are excellent for target molecules with RSD less than 1 and 5\%, 

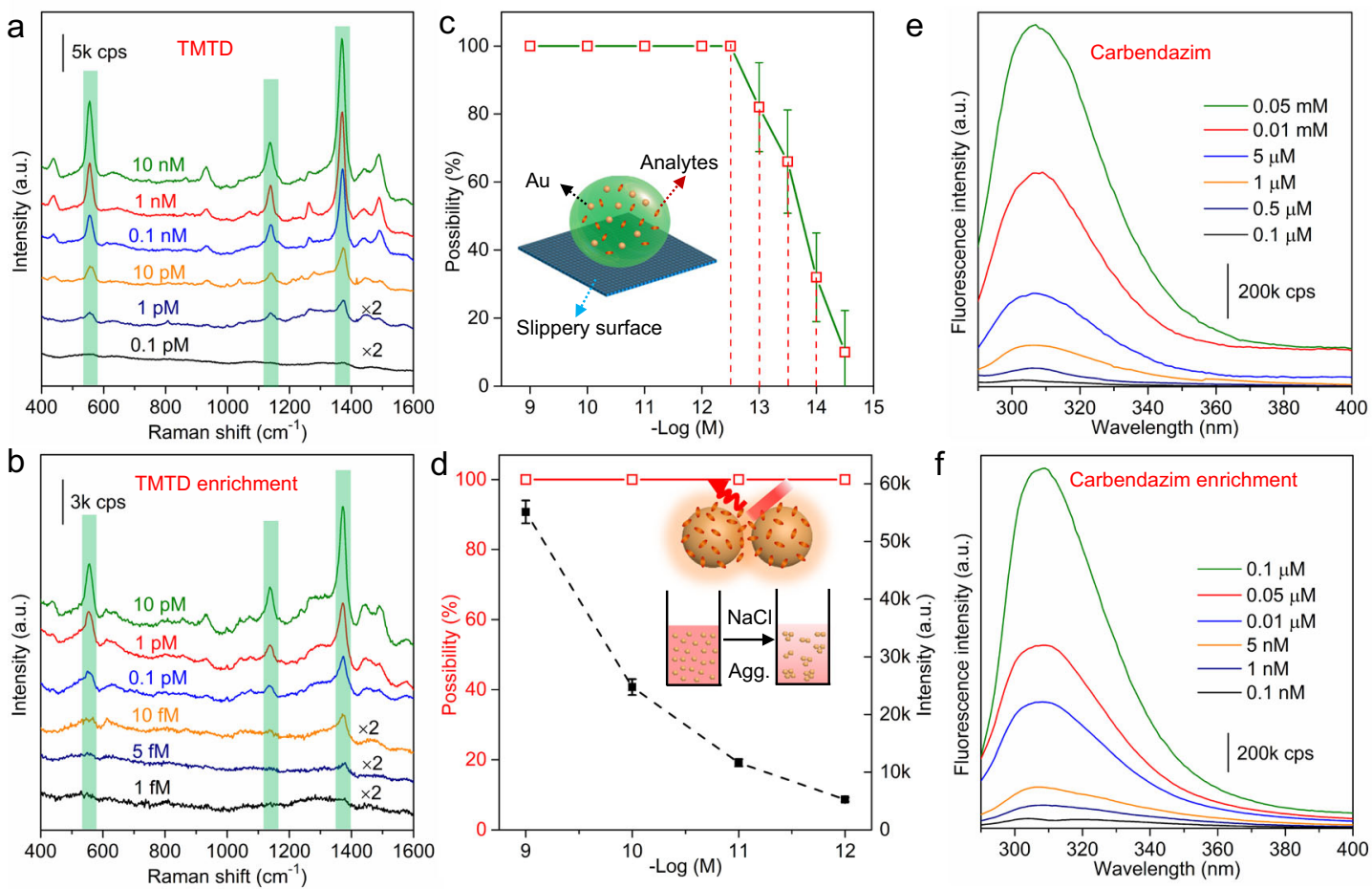

Fig. 4 Application in Raman and fluorescence detection by this enrichment strategy. Enhanced Raman spectra of TMTD a before and $\mathbf{b}$ after enrichment process of MN-PCDP. c Probability of SERS signals with different concentrations of TMTD after enrichment process of MN-PCDP by hydrophobic slippery SERS platform. The inserted schematic diagram shows hydrophobic slippery SERS platform. The data are reported as the average enrichment of five experiments. Error bars mean standard deviations. Shaded areas mean the characteristic peaks (555, 1138, and $1370 \mathrm{~cm}^{-1}$ ) of TMTD molecule. d Detection probability (red) and intensity of SERS signals (black) with different concentrations of TMTD after enrichment process of MN-PCDP by aggregating approach with Au colloid. The inserted schematic diagram shows aggregating approach with Au colloid. The data are reported as the average enrichment of five experiments. Error bars mean standard deviations. Fluorescence spectra of carbendazim $\mathbf{e}$ before and $\mathbf{f}$ after the enrichment of MN-PCDP.

\section{Table 1 Detectability of the MNPs for organic pollutants reported in the literature.}

\begin{tabular}{|c|c|c|c|}
\hline Types of MNPs ${ }^{a}$ & Analyte & LOD & Ref. \\
\hline MN-PCDPb & TMTD & $5 \times 10^{-15} \mathrm{M}$ & This work \\
\hline $\begin{array}{l}\mathrm{Fe}_{3} \mathrm{O}_{4} @ \mathrm{SiO}_{2} @ \mathrm{Ag} \\
\text { nanospindles }\end{array}$ & TMTD & $10^{-7} \mathrm{M}$ & \\
\hline $\mathrm{Fe}_{3} \mathrm{O}_{4} @ \mathrm{Au} \mathrm{NRs}$ array & TMTD & $10^{-9} \mathrm{M}$ & 44 \\
\hline $\begin{array}{l}\mathrm{Fe}_{3} \mathrm{O}_{4} @ \mathrm{SiO}_{2} @ \mathrm{Ag} \text { core- } \\
\text { shell MNPs }\end{array}$ & TMTD & $10^{-9} \mathrm{M}$ & 45 \\
\hline $\begin{array}{l}\text { Cube-like } \\
\mathrm{Fe}_{3} \mathrm{O}_{4} @ \mathrm{SiO}_{2} @ \mathrm{Au} @ \mathrm{Ag}\end{array}$ & TMTD & $5 \times 10^{-11} \mathrm{M}$ & 46 \\
\hline $\begin{array}{l}\text { Flower-like } \\
\mathrm{Fe}_{3} \mathrm{O}_{4} @ \mathrm{SiO}_{2} @ \mathrm{Ag}\end{array}$ & TMTD & $10^{-11} \mathrm{M}$ & 47 \\
\hline $\mathrm{Fe}_{3} \mathrm{O}_{4} @ \mathrm{Ag}-\mathrm{PEI}-\mathrm{Au} @ \mathrm{Ag}$ & TMTD & $5 \times 10^{-12} \mathrm{M}$ & 48 \\
\hline $\mathrm{Au} @ \mathrm{Fe}_{3} \mathrm{O}_{4}$ network & TMTD & $5 \times 10^{-14} \mathrm{M}$ & 49 \\
\hline MN-PCDP & Diquat & $10^{-12} \mathrm{M}$ & This work \\
\hline $\mathrm{Fe}_{3} \mathrm{O}_{4} @$ AuNRs assemblies & Diquat & $10^{-9} \mathrm{M}$ & \\
\hline $\mathrm{Fe}_{3} \mathrm{O}_{4} @ \mathrm{Ag}-\mathrm{PEI}-\mathrm{Au} @ \mathrm{Ag}$ & Paraquat & $10^{-10} \mathrm{M}$ & 48 \\
\hline$M N-P C D P$ & Carbendazim & $5 \times 10^{-12} \mathrm{M}$ & This work \\
\hline $\mathrm{Au} / \mathrm{Fe}_{3} \mathrm{O}_{4}$ nanocomposite & Carbendazim & $2.3 \times 10^{-9} \mathrm{M}$ & \\
\hline MN-PCDP & BPA & $10^{-10} \mathrm{M}$ & This work \\
\hline Magnetic gold nanoclusters & BPA & $10^{-9} \mathrm{M}$ & \\
\hline $\begin{array}{l}\text { Magnetic-bead biosensing } \\
\text { platform }\end{array}$ & BPA & $4.3 \times 10^{-10} \mathrm{M}$ & 52 \\
\hline MN-PCDP & Anthracene & $10^{-9} \mathrm{M}$ & This work \\
\hline $\mathrm{Fe}_{3} \mathrm{O}_{4} @ \mathrm{Ag} \mathrm{MNPs}$-thiol & Anthracene & $10^{-6} \mathrm{M}$ & \\
\hline
\end{tabular}

respectively. The Raman detectable reproducibility of TMTD by hydrophobic slippery SERS platform is shown in Supplementary Figs. 24-30. In Fig. 4c, the Raman signals of TMTD characteristic peaks are acquired with $100 \%$ detection probability in $0.5 \mathrm{pM}$, $\sim 65 \%$ in $50 \mathrm{fM}$, and $\sim 10 \%$ in $5 \mathrm{fM}$. In addition, the solution-based aggregation approach, a simplest and effective way in commercial detection platforms at present, is adopted to clarify the consistency of SERS signal. As shown in Fig. 4d and Supplementary Figs. 31, 32, the SERS signals display superior spectral reproducibility and uniformity with a $100 \%$ detection probability and RSD value of $\sim 5 \%$, even at TMTD concentration of $10^{-12} \mathrm{M}$. In Fig. 4e, f and Supplementary Fig. 33, using the current enrichment-typed sensing strategy, the LODs of fluorescence detections for the concentrated and enriched molecules of carbendazim and BPA are also enhanced by $2-3$ orders of magnitude. In this study, the enrichment protocol based on the adsorption and desorption processes of MN-PCDP adsorbent may significantly increase the sensitivity of plasmonic sensors, compared with the LOD for the same molecules with different SERS and fluorescence detection protocols (Table 1) $31-33$. Thus, the current strategy has wider applicability for mass spectrometry, chromatography, and other detection protocols.

Separation and selective enrichment in real-sample complex matrix via current strategy. Based on the distinguishing and selective absorption capacity for different molecules (Fig. 2 and Supplementary Figs. 10, 11 $)^{29,34}$, the mesoporous nanosponge is expected to be used in the separation of interested molecules from 

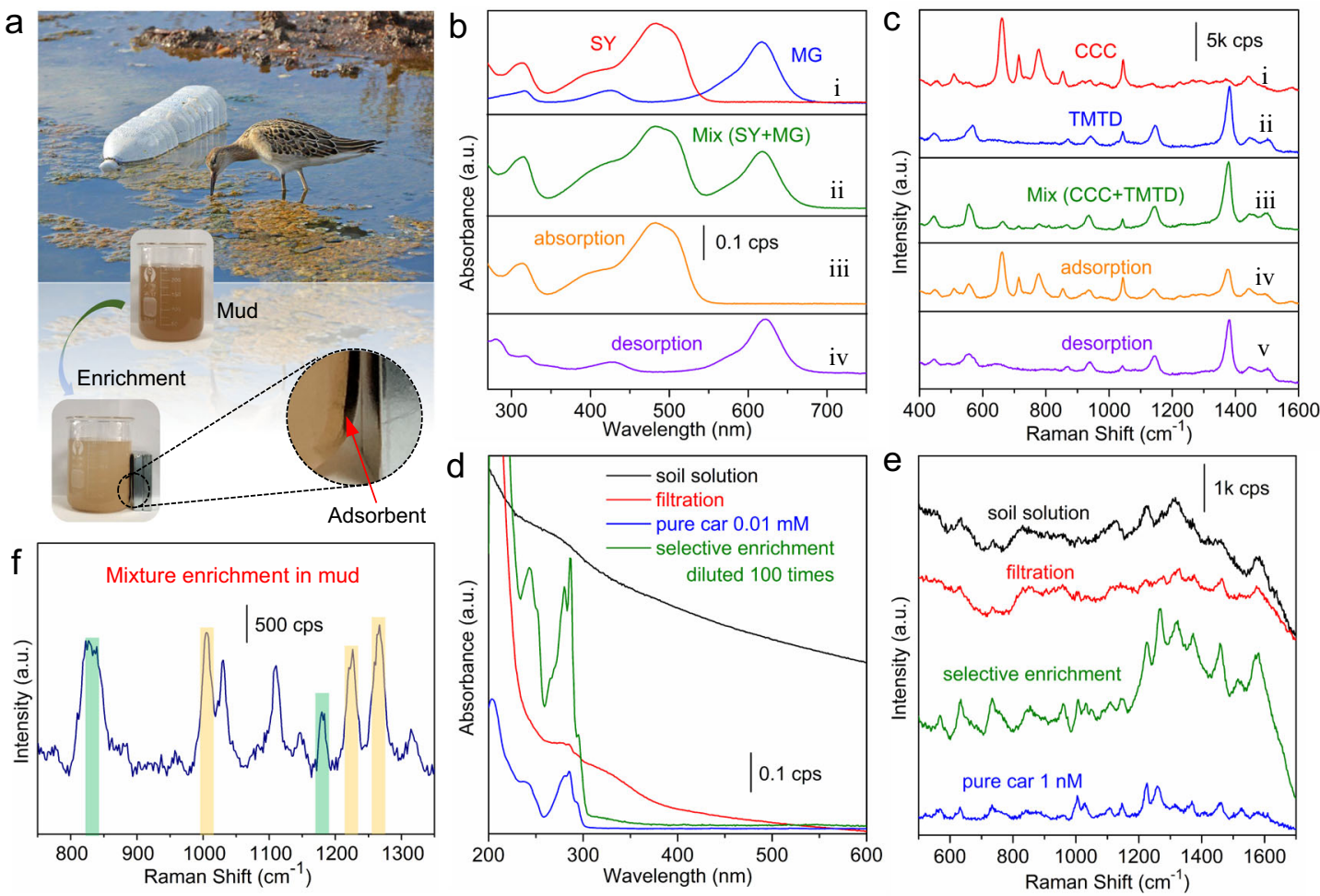

Fig. 5 Separation and selective enrichment in complex matrix used this strategy. a Optical photographs about the enrichment process of MN-PCDP in mud water. $\mathbf{b} U V-V$ is spectra of SY $(\mathbf{i}, 0.01 \mathrm{mM}), M G(\mathbf{i}, 0.01 \mathrm{mM})$ and SY/MG (ii, both with $0.01 \mathrm{mM})$ mixture solution. iii The filtration mixture solution after the absorption of MN-PCDP for MG. iv The desorption solution of captured molecules (MG) redispersed in ethanol from the cavity of MN-PCDP adsorbent. c Raman spectra of CCC (i, $0.1 \mathrm{mM})$, TMTD (ii, $1 \mu \mathrm{M})$ and iii CCC $(0.1 \mathrm{mM}) / \operatorname{TMTD}(1 \mu \mathrm{M})$ mixture solution before and after iv absorption, $\mathbf{v}$ desorption. $\mathbf{d}$ UV -Vis spectra about selective enrichment of organic pollutant molecules (carbendazim, $0.01 \mathrm{mM}$ ) from practical samples. e Raman spectra about selective enrichment of organic pollutant molecules (carbendazim, $1 \mathrm{nM}$ ) from practical samples. f Raman spectrum of mixture (BPA and carbendazim) after the enrichment process of MN-PCDP in real samples. Shaded areas mean the characteristic peaks of BPA (green, 830 and $1179 \mathrm{~cm}^{-1}$ ) and carbendazim (yellow, 1004, 1222, and $1263 \mathrm{~cm}^{-1}$ ) molecules.

the mixed systems. As shown in Fig. $5 \mathrm{~b}$ and Supplementary Fig. 34, the malachite green (MG) and sunset yellow (SY) molecules are firstly mixed together and became to be the mixture solution (MG + SY) (Fig. 5b-i-ii). Then, after adsorption and separation processes using MN-PCDP, clearly, there is only SY left in the mixture solution (Fig. 5b-iii). Similarly, with a desorption process, the MG molecule is also successfully separated (Fig. 5b-iv). Following, two different pesticide molecules, namely chlormequat chloride (CCC) and TMTD, are used to further evaluate the selective separation using SERS detection (Fig. 5c-iiii). Obviously, once TMTD is captured and separated, the SERS signals of CCC in the mixture solution significantly increases (Fig. $5 \mathrm{c}$-iv), indicating that most TMTD molecules have been adsorbed. Moreover, the single Raman (Fig. 5c-v) and UV-Vis spectra (Supplementary Fig. 35) from TMTD molecules can be observed, illustrating only TMTD is effectively selected and separated by MN-PCDP.

Another advantage of the current enrichment protocol is that the interference of complex matrix can be effectively eliminated in the detection of real-sample system ${ }^{35-37}$. After the adsorption process, the MN-PCDP adsorbent can be easily separated by a magnet from a complicated environment containing, e.g., mud and microorganism (Fig. 5a). Figure 5d exhibits the UV-Vis spectra of carbendazim at a concentration of $0.01 \mathrm{mM}$ in the filtered soil solution. It can be observed that the characteristic absorption peaks of carbendazim are very weak even after filtration. However, using the current selective separation and enrichment protocol, the absorption peaks intensity of carbendazim (Fig. 5d), BPA and MG (Supplementary Fig. 36a, b) are respectively increased 463,550 , and 516 times comparing with pure solution. Similarly, as shown in Fig. 5e, when the concentration of detection molecules goes down to $1 \mathrm{nM}$, the SERS signals of carbendazim molecule in practical soil solution samples even after filtration are almost unobservable, whereas they can be easily detected after selective enrichment by $\mathrm{MN}$ PCDP. The intensities of Raman characteristic peak from BPA and MG (Supplementary Fig. 37a, b) in complex matrix are also greatly improved.

In addition, in the real environment, more than one molecule is always studied, thus it is very important to realize the simultaneous detection of multiple molecules, especially in the existence of matrix interference. Figure $5 f$ reveals that both the characteristic peaks of BPA $\left(830\right.$ and $\left.1179 \mathrm{~cm}^{-1}\right)$ and carbenda$\operatorname{zim}\left(1004,1222\right.$, and $\left.1263 \mathrm{~cm}^{-1}\right)$ evidently appear in the Raman spectra of mixture solution, e.g., $1 \mu \mathrm{M}$ BPA and $10 \mathrm{nM}$ carbendazim, indicating the great absorption and detection capability for different molecules at the same time. Furthermore, the MN-PCDP demonstrates a superior reusability. As shown in Supplementary Fig. 38, six consecutive BPA adsorption/desorption cycles are performed and the regenerated MN-PCDP exhibited almost inappreciable decrease $(90.2-84.3 \%)$ in performance compared to the as-synthesized polymer.

\section{Discussion}

In summary, we have developed a robust and efficient sensing strategy based on the MN-PCDP mesoporous nanoponges to capture and enrich organic pollutants from water. In this strategy, the MN-PCDP adsorbent exhibits excellent selective adsorption 
and enrichment capacity for various kinds of pollutants, eliminating the interference of complex matrix in the real-sample environments. Meanwhile, in diverse detection protocols of organic pollutants, e.g., UV-Vis, Raman, and fluorescent, the current sensing strategy significantly may increase the sensitivity with $2-3$ orders of magnitude. Moreover, using the immobilization of magnetic NPs, the adsorption, separation, and enrichment processes by MN-PCDP can be completed within $2-3 \mathrm{~min}$. Therefore, the current robust sensing strategy with the ultrarapid, selective, and highly efficient molecule enrichment capability is believed to be applicable to a wider range of sensing devices for a cost-effective, simple, fast, flexible and portable detection. In the future, single-particle-MN-PCDP combining with $\mathrm{Au}$ NPs (SERS substrate) could dramatically lower the detection limit and enables higher spatial and temporal resolution ${ }^{38-42}$, thus build single NP sensor to improve detection sensitivity (Supplementary Fig. 39).

\section{Methods}

Preparation of magnetite NPs $\left(\mathrm{Fe}_{3} \mathrm{O}_{4}\right)$. The carboxyl-functionalized magnetite NPs $\left(\mathrm{Fe}_{3} \mathrm{O}_{4}\right)$ with highly water-dispersibility were synthesized by a modified solvothermal reaction approach ${ }^{22}$. Typically, $\mathrm{FeCl}_{3} \cdot 6 \mathrm{H}_{2} \mathrm{O}(1.08 \mathrm{~g}, 4.0 \mathrm{mmol})$ and trisodium citrate $(0.20 \mathrm{~g}, 0.68 \mathrm{mmol})$ were dissolved in ethylene glycol $(20 \mathrm{~mL})$ with stirring at $500 \mathrm{rpm}$. Afterward, sodium acetate trihydrate $(2.0 \mathrm{~g}, 15 \mathrm{mmol})$ was added and the mixture was stirred for $30 \mathrm{~min}$. Then, the mixture was sealed in a Teflon-lined stainless-steel autoclave $(50 \mathrm{~mL})$. The autoclave was heated at $200^{\circ} \mathrm{C}$ for $12 \mathrm{~h}$, and then allowed to cool to room temperature. The black products were washed with ethanol and ultrapure water for several times. Finally, the carboxylfunctionalized magnetite $\mathrm{NPs}\left(\mathrm{Fe}_{3} \mathrm{O}_{4}\right)$ were separated by magnet, re-dispersed in ethanol, and dried in vacuum drying oven at $30^{\circ} \mathrm{C}$.

Preparation of magnetic NPs immobilized porous $\beta$-CD polymer (MN-PCDP). The MN-PCDP composites were then prepared by modification of nucleophilic aromatic substitution method of hydroxyl groups of $\beta-\mathrm{CD}^{20}$. A dried $100 \mathrm{~mL}$ Shrek reaction vial with a magnetic stir bar was charged with $\beta-C D(0.82 \mathrm{~g}, 0.724 \mathrm{mmol})$, TFT $(0.40 \mathrm{~g}, 1.03 \mathrm{mmol})$, and $\mathrm{K}_{2} \mathrm{CO}_{3}(1.28 \mathrm{~g}, 9.28 \mathrm{mmol})$ and dried $\mathrm{Fe}_{3} \mathrm{O}_{4}(0.041 \mathrm{~g})$. The vial was flushed with $\mathrm{N}_{2}$ gas for $10 \mathrm{~min}$, then an anhydrous THF/DMF mixture $(9: 1 \mathrm{v} / \mathrm{v}, 40 \mathrm{~mL})$ was added and the vial was purged with $\mathrm{N}_{2}$ for an additional $5 \mathrm{~min}$. After that, the $\mathrm{N}_{2}$ inlet was removed. The mixture was stirred at $500 \mathrm{rpm}$ and refluxed at $85^{\circ} \mathrm{C}$ for $36 \mathrm{~h}$ under nitrogen protection. The brown suspension was cooled to room temperature and magnetically separated the supernatant by magnet. The precipitate was washed twice with an appropriate amount of distilled water, THF, ethanol, and $\mathrm{CH}_{2} \mathrm{Cl}_{2}$, respectively. The final precipitate was vacuum dried at $77 \mathrm{~K}$ in a liquid nitrogen bath for $24 \mathrm{~h}$ and then the magnetic NPs immobilized porous $\beta$-CD polymer (MN-PCDP) was obtained.

Batch adsorption kinetic studies. In studies, the dried polymer (MN-PCDP, $20 \mathrm{mg}$ ) was initially washed with $\mathrm{H}_{2} \mathrm{O}$ for 2 times and then separated by a magnet. Adsorption kinetic studies for different pollutants were performed in $30 \mathrm{~mL}$ scintillation vials with $20 \mathrm{~mL}$ organic pollutant solution and $20 \mathrm{mg}$ adsorbent, at ambient temperature on a hot plate at $25^{\circ} \mathrm{C}$. Then the sample was shaken at $250 \mathrm{rpm}$ until the adsorption equilibrium was reached. The mixture was immediately stirred and $1 \mathrm{~mL}$ aliquots of the suspension were taken at certain intervals via syringe and filtered immediately by a $0.22 \mu \mathrm{m}$ PTFE membrane filter. The residual concentration of the pollutant in each sample was determined by UV-vis spectroscopy.

Calculation of removal efficiency. The removal efficiency of pollutant removal by the adsorbent was determined by the following equation:

$$
\text { Removal efficiency }(\%)=\frac{C_{0}-C_{t}}{C_{0}} \times 100
$$

where $C_{0}$ and $C_{t}$ are the initial and residual concentration of pollutant in the stock solution and filtrate, respectively.

Flow-through adsorption experiments. Individual pollutants were at high concentrations $(\mathrm{mM}) .5 .0 \mathrm{mg}$ of the MN-PCDP adsorbent was washed with deionized $\mathrm{H}_{2} \mathrm{O}$ for 2 times, then the precipitate was pushed by a syringe through a $0.22 \mu \mathrm{m}$ PTFE membrane filter to form a thin layer of the adsorbent on the filter membrane. $5 \mathrm{~mL}$ of the pollutant stock solution was then pushed through the adsorbent in $\sim 30 \mathrm{~s}\left(10 \mathrm{~mL} \mathrm{~min}^{-1}\right.$ flow rate). The filtrate was then measured by UV-Vis spectroscopy to determine the pollutant removal efficiency.
MN-PCDP desorption studies. $100.0 \mathrm{mg}$ of the adsorbent was washed with deionized $\mathrm{H}_{2} \mathrm{O}$ for 2 times, and then added to the organic pollutant stock solution $(0.01 \mathrm{mM})$ with determined volume $(100,250$, and $500 \mathrm{~mL})$. The mixture was shaken at $250 \mathrm{rpm}$ for $1 \mathrm{~min}$ at $25^{\circ} \mathrm{C}$. After separating the supernatant and the adsorbent by an external magnet, the supernatant was filtered through a $0.22 \mu \mathrm{m}$ filter membrane and determined by UV-Vis spectroscopy. Meanwhile, the precipitate was evaporated to dryness with a gentle nitrogen stream, then the residue was dissolved in $1 \mathrm{~mL}$ of ethanol to desorb the adsorbed organic pollutant. The desorption solution was measured by UV-Vis spectroscopy and compared with the initial concentration of pollutant in the stock solution.

Calculation of enrichment efficiency. The enrichment efficiency of pollutant adsorbed by the adsorbent was determined by the following equation:

$$
\text { Enrichment efficiency }=\frac{C}{C_{0}} \text {. }
$$

Where $C_{0}$ and $C$ are the initial and desorbed solution concentration of pollutant respectively.

Fluorescence measurement. The fluorescence spectra of pure solution were directly measured by a fluorescence spectrophotometer.

Preparation of SERS active Au NPs. The Au NPs with different sizes in diameter were synthesized based on a modified citrate reduction approach. The growth process of gold NPs with different sizes included three steps. For step $1,100 \mathrm{~mL}$ of ultrapure water was added into a conical flask and heated to boiling. Then, $4 \mathrm{ml}$ of $1 \mathrm{wt} \%$ sodium citrate (SC) solution was injected immediately, and $3.2 \mathrm{~mL}$ of $10 \mathrm{mM}$ $\mathrm{HAuCl}_{4}$ was added after $3 \mathrm{~min}$. Kept the reaction for $25 \mathrm{~min}$ and made it natural cooling, then the Au seeds were obtained. For step $2,80 \mathrm{~mL}$ of ultrapure water and $20 \mathrm{~mL}$ of Au seeds were mixed into the conical flask and heated to boiling. Then, $2 \mathrm{~mL}$ of $1 \mathrm{wt} \%$ sodium citrate solution was injected immediately, and $0.2 \mathrm{~mL}$ of $\mathrm{HAuCl}_{4}$ was added $3 \mathrm{~min}$ later. Then additional $0.2 \mathrm{~mL} \times 9$ dosage of $\mathrm{HAuCl}_{4}$ was injected every $8 \mathrm{~min}$. After the last precursor was added, the reaction was kept for $25 \mathrm{~min}$, and Au NPs-25 nm were obtained. For step 3, Au NPs prepared in step 2 were used as the seed solution, and the growth process was repeated as growth steps 2, and then Au NPs-55 nm were obtained in this step.

SERS measurement. SERS measurement is based on the hydrophobic slippery surface ${ }^{17}$. Concentrated molecules and Au NPs were prepared on a hydrophobic slippery Teflon membrane as follows: First, a Teflon membrane was attached on a flat glass slide $(5 \mathrm{~cm} \times 5 \mathrm{~cm})$ by using a double-sided adhesive. Then, $0.5 \mathrm{~mL}$ of perfluorinated fluid was dispersed by spin coating. The low speed was $300 \mathrm{rpm}$ for $30 \mathrm{~s}$, and the high speed was $1500 \mathrm{rpm}$ for $1 \mathrm{~min}$. After the excess lubricating liquid was removed by centrifugal force, and the infused membrane was heated for 30 min. Lastly, $50 \mu \mathrm{L}$ of probe molecules and $10 \mu \mathrm{L}$ of Au colloids were simultaneously dropped onto the slippery surface. During drying, the contact line shrunk because of the low friction of the lubricated Teflon surface. As a result, the initial droplet could be concentrated into a small area less than $0.5 \mathrm{~mm}$ in diameter.

\section{Data availability}

The data that support the findings of this study are available within the paper and its Supplementary Information or from the corresponding authors on reasonable request.

Received: 13 December 2020; Accepted: 19 October 2021; Published online: 25 November 2021

\section{References}

1. Kelly, B. C., Ikonomou, M. G., Blair, J. D., Morin, A. E. \& Gobas, F. A. P. C. Food web-specific biomagnification of persistent organic pollutants. Science 317, 236-239 (2007).

2. Schwarzenbach, R. P. et al. The challenge of micropollutants in aquatic systems. Science 313, 1072-1077 (2006).

3. Jones, K. C. \& Voogt, P. De. Persistent organic pollutants (POPs): state of the science. Environ. Pollut. 100, 209-221 (1999).

4. $\mathrm{Pi}, \mathrm{Y}$. H. et al. Adsorptive and photocatalytic removal of persistent organic pollutants (POPs) in water by metal-organic frameworks (MOFs). Chem. Eng. J. 337, 351-371 (2018).

5. Xu, H. X., Bjerneld, E. J., Käll, M. \& Börjesson, L. Spectroscopy of single hemoglobin molecules by surface enhanced Raman scattering. Phys. Rev. Lett. 83, 4357-4360 (1999).

6. Kneipp, K. ; et al. Single molecule detection using surface-enhanced Raman scattering (SERS). Phys. Rev. Lett. 78, 1667-1670 (1997).

7. Nie, S. M. \& Emory, S. R. Probing single molecules and single nanoparticles by surface-enahnced Raman scattering. Science 275, 1102-1106 (1997). 
8. Xu, H. X., Aizpurua, J., Kall, M. \& Apell, P. Electromagnetic contributions to single-molecule sensitivity in surface-enhanced Raman scattering. Phys. Rev. E 62, 4318 (2000).

9. Li, J. F. et al. Shell-isolated nanoparticle-enhanced Raman spectroscopy. Nature 464, 392-395 (2010).

10. Panneerselvam, R. et al. Surface-enhanced Raman spectroscopy: bottlenecks and future directions. Chem. Commun. 54, 10-25 (2017)

11. Otto, A., Mrozek, I., Grabhorn, H. \& Akemann, W. Surface-enhanced Raman scattering. J. Phys.-Condens. Matter 4, 1143-1212 (1992).

12. Schatz \& George, C. Theoretical studies of surface enhanced Raman scattering. Acc. Chem. Res. 17, 370-376 (1984).

13. Schlucker, S. Surface-enhanced Raman spectroscopy: concepts and chemical applications. Angew. Chem. Int. Ed. Engl. 53, 4756-4795 (2014).

14. Moskovits, M. Surface-enhanced Raman spectroscopy: a brief retrospective. J. Raman Spectrosc. 36, 485-496 (2005).

15. Zeman, E. J. \& Schatz, G. C. An accurate electromagnetic theory study of surface enhancement factors for silver, gold, copper, lithium, sodium, aluminum, gallium, indium, zinc, and cadmium. J. Phys. Chem. 91, 634-643 (1987).

16. Guo, X. T. et al. Molecular imprinting-based surface-enhanced Raman scattering sensors. ACS Sens. 5, 601-619 (2020).

17. Zhang, D. J. et al. Buoyant particulate strategy for few-to-single particle-based plasmonic enhanced nanosensors. Nat. Commun. 11, 2603 (2020).

18. Hao, R., You, H. J., Zhu, J., Chen, T. \& Fang, J. X. “Burning Lamp”-like robust molecular enrichment for ultrasensitive plasmonic nanosensors. ACS Sens. 5, 781-788 (2020).

19. De Angelis, F. et al. Breaking the diffusion limit with super-hydrophobic delivery of molecules to plasmonic nanofocusing SERS structures. Nat. Photonics 5, 682-687 (2011).

20. Alsbaiee, A. et al. Rapid removal of organic micropollutants from water by a porous beta-cyclodextrin polymer. Nature 529, 190-194 (2016).

21. Xiao, L. L. et al. Beta-cyclodextrin polymer network sequesters perfluorooctanoic acid at environmentally relevant concentrations. J. Am. Chem. Soc. 139, 7689-7692 (2017).

22. Liu, J. et al. Highly water-dispersible biocompatible magnetite particles with low cytotoxicity stabilized by citrate groups. Angew. Chem. Int. Ed. 48, 5875-5879 (2009).

23. Jiang, H. L. et al. A novel crosslinked $\beta$-cyclodextrin-based polymer for removing methylene blue from water with high efficiency. Colloid Surf. A 560, 59-68 (2019).

24. Xu, G. Z. et al. Simple synthesis of a swellable porous $\beta$-cyclodextrin-based polymer in the aqueous phase for the rapid removal of organic micropollutants from water. Green. Chem. 21, 6062-6072 (2019).

25. Huang, Q. Y., Chai, K. G., Zhou, L. Q. \& Ji, H. B. A phenyl-rich $\beta$-cyclodextrin porous crosslinked polymer for efficient removal of aromatic pollutants: insight into adsorption performance and mechanism. Chem. Eng. J. https:// doi.org/10.1016/j.cej.2020.124020 (2020).

26. Wang, Z. H., Zhang, P. B., Hu, F., Zhao, Y. F. \& Zhu, L. P. A crosslinked betacyclodextrin polymer used for rapid removal of a broad-spectrum of organic micropollutants from water. Carbohyd. Polym. 177, 224-231 (2017).

27. Manakker, F. V. D., Vermonden, T., Nostrum, C. F. V. \& Hennink, W. E. Cyclodextrin-based polymeric materials: synthesis, properties, and pharmaceutical/biomedical applications. Biomacromolecules 10, 3157-3175 (2009).

28. Wankar, J. et al. Recent advances in host-guest self-assembled cyclodextrin carriers: implications for responsive drug delivery and biomedical engineering. Adv. Funct. Mater. 30, 1909049 (2020).

29. Zhao, R., Wang, Y., Li, X., Sun, B. \& Wang, C. Synthesis of $\beta$-cyclodextrinbased electrospun nanofiber membranes for highly efficient adsorption and separation of methylene blue. ACS Appl. Mater. Interfaces 7, 26649-26657 (2015).

30. Zhang, D. J. et al. Hydrophobic slippery surface-based surface-enhanced raman spectroscopy platform for ultrasensitive detection in food safety applications. Anal. Chem. 91, 4687-4695 (2019).

31. Chen, X. et al. Detection and quantification of carbendazim in Oolong tea by surface-enhanced Raman spectroscopy and gold nanoparticle substrates. Food Chem. 293, 271-277 (2019).

32. Zhu, X. Y. et al. A novel graphene-like titanium carbide MXene/Au-Ag nanoshuttles bifunctional nanosensor for electrochemical and SERS intelligent analysis of ultra-trace carbendazim coupled with machine learning. Ceram. Int. https://doi.org/10.1016/j.ceramint.2020.08.121 (2020).

33. Zhai, Y. et al. Metal-organic-frameworks-enforced surface enhanced Raman scattering chip for elevating detection sensitivity of carbendazim in seawater. Sensors Actuat. B-Chem. https://doi.org/10.1016/j.snb.2020.128852 (2021).

34. Jiang, H. L. et al. Selective adsorption of anionic dyes from aqueous solution by a novel $\beta$-cyclodextrin-based polymer. J. Mol. Struct. 1203, 127373 (2019).

35. Song, D. et al. A label-free SERRS-based nanosensor for ultrasensitive detection of mercury ions in drinking water and wastewater effluent. Anal. Methods 9, 154-162 (2017).
36. Bao, Z. Y., Dai, J., Lei, D. Y. \& Wu, Y. Maximizing surface-enhanced Raman scattering sensitivity of surfactant-free $\mathrm{Ag}-\mathrm{Fe}_{3} \mathrm{O}_{4}$ nanocomposites through optimization of silver nanoparticle density and magnetic self-assembly. $J$. Appl. Phys. 114, 124305 (2013).

37. Bao, Z. Y. et al. Quantitative SERS detection of low-concentration aromatic polychlorinated biphenyl-77 and 2,4,6-trinitrotoluene. J. Hazard. Mater. 280, 706-712 (2014).

38. $\mathrm{Li}, \mathrm{Q}$. et al. Detection of single proteins with a general nanopore sensor. ACS Sens. 4, 1185-1189 (2019).

39. Lu, S. M. et al. Electrochemical sensing at a confined space. Anal. Chem. 92, 5621-5644 (2020)

40. Ying, Y. L. et al. Electrochemical confinement effects for innovating new nanopore sensing mechanisms. Small Methods 2, 1700390 (2018).

41. Shi, L. et al. Plasmon resonance scattering spectroscopy at the single nanoparticle level: real-time monitoring of a click reaction. Angew. Chem. Int. Ed. 52, 6011-6014 (2013).

42. Gao, Q. et al. Highly sensitive impedimetric sensing of DNA hybridization based on the target DNA-induced displacement of gold nanoparticles attached to ssDNA probe. Electrochem. Commun. 13, 335-337 (2013).

43. $\mathrm{He}, \mathrm{Q}$. et al. Fabrication of $\mathrm{Fe}_{3} \mathrm{O}_{4} @ \mathrm{SiO}_{2} @ \mathrm{Ag}$ magnetic-plasmonic nanospindles as highly efficient SERS active substrates for label-free detection of pesticides. N. J. Chem. 41, 1582-1590 (2017).

44. Tang, S. Y. et al. Efficient enrichment and self-assembly of hybrid nanoparticles into removable and magnetic SERS substrates for sensitive detection of environmental pollutants. ACS Appl. Mater. Interfaces $\mathbf{9}$, 7472-7480 (2017).

45. Wang, C. W. et al. Seed-mediated synthesis of high-performance silver-coated magnetic nanoparticles and their use as effective SERS substrates. Colloids Surf. A: Physicochem. Eng. Asp. 506, 393-401 (2016).

46. Sun, M. et al. Cube-like $\mathrm{Fe}_{3} \mathrm{O}_{4} @ \mathrm{SiO}_{2} @ \mathrm{Au} @ \mathrm{Ag}$ magnetic nanoparticles: a highly efficient SERS substrate for pesticide detection. Nanotechnology 29 , 165302 (2018).

47. Wang, C. W. et al. Sonochemical synthesis of highly branched flower-like $\mathrm{Fe}_{3} \mathrm{O}_{4} @ \mathrm{SiO}_{2} @ \mathrm{Ag}$ microcomposites and their application as versatile SERS substrates. Nanoscale 8, 19816-19828 (2016).

48. Wang, C. W. et al. Polyethylenimine-interlayered core-shell-satellite 3D magnetic microspheres as versatile SERS substrates. Nanoscale 7 , 18694-18707 (2015)

49. Yang, T. X. et al. Au dotted magnetic network nanostructure and its application for on-site monitoring femtomolar level pesticide. Small 10, 1325-1331 (2014)

50. $\mathrm{Li}$, Q. et al. A gold $/ \mathrm{Fe}_{3} \mathrm{O}_{4}$ nanocomposite for use in a surface plasmon resonance immunosensor for carbendazim. Microchim. Acta 186, 313 (2019).

51. Kadasala, N. R. \& Wei, A. Trace detection of tetrabromobisphenol A by SERS with DMAP-modified magnetic gold nanoclusters. Nanoscale 7, 10931-10935 (2015).

52. Xiao, R., Wang, W. C., Zhu, A. N. \& Long, F. Single functional magnetic-bead as universal biosensing platform for trace analyte detection using SERSnanobioprobe. Biosens. Bioelectron. 79, 661-668 (2016).

53. Du, J. J. \& Jing, C. Y. Preparation of thiol modified $\mathrm{Fe}_{3} \mathrm{O}_{4} @ \mathrm{Ag}$ magnetic SERS probe for PAHs detection and identification. J. Phys. Chem. C 115, 17829-17835 (2011)

\section{Acknowledgements}

This work was supported by the programs supported by the National Natural Science Foundation of China (No. 21675122, 21874104, 22074115 awarded to J.X.F. and 62022001 awarded to D.Y.L.), the Key Research Program in Shaanxi (2017NY-114 awarded to J.X.F.), and Natural Science Foundation of Shaanxi Province (No. 2019JLP-19 awarded to H.J.Y.), the World-Class Universities (Disciplines, awarded to J.X.F.) and the Characteristic Development Guidance Funds for the Central Universities (awarded to J.X.F.). The characterizations of materials are supported by the Instrument Analysis Center of Xi'an Jiaotong University.

\section{Author contributions}

L.L.Z. synthesized the materials, carried out the characterizations and performance, analyzed the data, and wrote the draft of the manuscript. Y.G., R.H., Y.F.S., H.N., Y.Z.D. D.J.L. and D.Y.L. contributed in part of the TEM, Raman, and fluorescence characterizations. J.X.F. supervised the project, designed the experiments, contributed in discussions, comments, and writing of the manuscript. H.J.Y. designed the partial experiments, contributed in discussions and comments. All authors discussed the results and commented on the manuscript.

\section{Competing interests}

The authors declare no competing of interest. 


\section{Additional information}

Supplementary information The online version contains supplementary material available at https://doi.org/10.1038/s41467-021-27100-2.

Correspondence and requests for materials should be addressed to Jixiang Fang.

Peer review information Nature Communications thanks the anonymous reviewer(s) for their contribution to the peer review of this work. Peer reviewer reports are available.

Reprints and permission information is available at http://www.nature.com/reprints

Publisher's note Springer Nature remains neutral with regard to jurisdictional claims in published maps and institutional affiliations. (c) (1) Open Access This article is licensed under a Creative Commons Attribution 4.0 International License, which permits use, sharing, adaptation, distribution and reproduction in any medium or format, as long as you give appropriate credit to the original author(s) and the source, provide a link to the Creative Commons license, and indicate if changes were made. The images or other third party material in this article are included in the article's Creative Commons license, unless indicated otherwise in a credit line to the material. If material is not included in the article's Creative Commons license and your intended use is not permitted by statutory regulation or exceeds the permitted use, you will need to obtain permission directly from the copyright holder. To view a copy of this license, visit http://creativecommons.org/ licenses/by/4.0/.

(C) The Author(s) 2021 